\title{
Experimental investigation of the overall Residence Time of Pebbles in a Pebble Bed Reactor (PBR) using Radioactive Pebble
}

\author{
Vaibhav Khane ${ }^{[1]}$, Mahmoud M. Taha ${ }^{[1]}$ and Muthanna H. Al-Dahhan ${ }^{[1,2]^{*}}$ \\ ${ }^{[1]}$ Department of Chemical and Biochemical Engineering, Missouri University of Science and \\ Technology, 110 Bertelsmeyer Hall, 1101 N State St., Rolla, MO, USA \\ ${ }^{[2]}$ Department of Mining and Nuclear Engineering, Missouri University of Science and \\ Technology, 222 Fulton Hall, 301 W. $14^{\text {th }}$ St., Rolla, MO, USA
}

\begin{abstract}
The granular flow of pebbles in a pebble bed reactor (PBR) under the influence of gravity is a dense granular flow with long-lasting frictional contacts. The basic governing physics is not fully understood and hence the dynamic core of a PBR and non-idealities associated with pebbles flow inside the reactor core are of non-trivial significance from the point of view of safety analyses, licensing, and thermal hydraulics. In the current study, overall and zonal pebbles residence time investigation is carried out by implementing noninvasive radioisotope-based flow visualization measurement techniques such as residence time distribution (RTD) and radioactive particle tracking (RPT). The characteristics of overall pebble residence time/transient number, zonal residence time, and the z-component of average zonal velocities at different initial seeding positions of a tracer particle have been summarized. It is found that the overall pebbles residence time/transient number increases (the z-component of average zonal velocities decreases) from the center towards the reactor wall. Also, pebbles' zonal residence time results (the whole core is divided into three zones) which provide more insight and understanding about PBR core dynamics have been reported. The benchmark data provided could be used for assessment of commercial/in-house computational methodologies related to granular flow investigations.
\end{abstract}

$\begin{array}{ll}\text { Key words: } & \text { Pebble bed reactor (PBR) } \\ & \text { Core dynamics } \\ & \text { Residence Time Distribution (RTD) } \\ & \text { Radioactive particle tracing (RPT) } \\ & \\ \text { * Corresponding author: Muthann H. Al-Dahhan } \\ \text { Email: aldahhanm@ @mst.edu }\end{array}$ 


\section{Introduction}

Nuclear energy will play a crucial role in achieving future global energy demands due to rapidly depleting fossil fuels, growing concerns about global warming and climate change issues, and sustainable development. Electricity generation by nuclear means is becoming more popular due to its zero greenhouse gas emissions. Nuclear energy is the only proven large-scale non fossil fuel source of energy and is capable of meeting rapidly increasing global energy demands. Current nuclear reactors in operation around the world fall under second or third generation systems, with most of the first-generation systems retired or revamped to second or third generation reactors. The fourth-generation (Gen IV) reactor designs are currently being researched around the world. A number of innovative reactor concepts were considered initially and six designs were finalized as Gen IV candidates. These designs meet the goals of the Gen IV initiative stated by the Generation IV International Forum (GIF). The main features of these designs are as follows: nuclear safety, higher resistance to proliferation of fissile materials, minimum radioactive waste generation, and efficient and economical design reducing the cost to build and operate such plants. These designs demand extensive research in order to prove their safety and reliability. The very high temperature reactor (VHTR) is one among these six designs that is using gaseous coolant. They are either prismatic block reactors or pebble bed reactors. The focus of this work is on pebble bed reactors.

The pebble bed reactor (PBR) concept was conceived and developed at Oak Ridge National Lab (ORNL). Nuclear fuel elements are in the form of TRISO (Tri-ISOtropic) fuel particles (Boer 2009) in which the uranium dioxide fuel particles are coated with four layers of carbon and silicon carbide. This TRISO coating acts as the primary containment of fission products. The fuel pebbles are mixed with graphite powder to form a fuel pebble with a diameter of $6 \mathrm{~cm}$ as shown in Figure 1. 

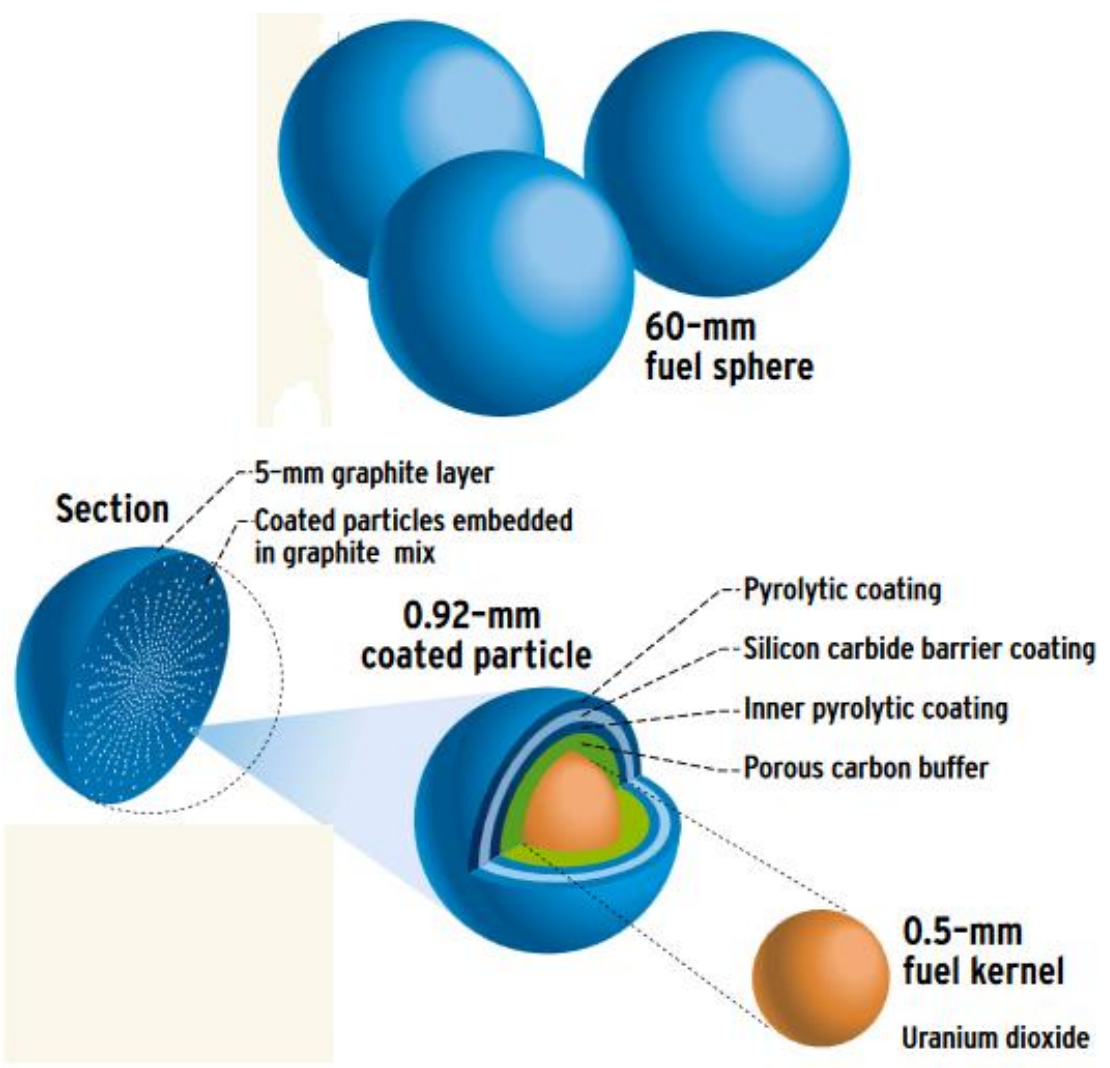

Figure 1: Fuel element design for PBR (taken from (Weil 2001))

The fuel pebbles move in the core under the influence of gravity (LaBar et al. 2004; Moses 2010) and continuously recirculated through the core and are monitored for burn-up (Curtius et al. 2015). After each pass through the reactor core, the fuel pebbles are examined to determine the amount of fissionable material left in them. If a pebble still contains certain usable amount of the fissile material, it is returned to the top of the reactor for a next pass. The returned radial placement position of a pebble depends on the fissile material content in that pebble. This continuous recirculation feature eliminates the need to shut down the reactor for refueling and hence an uninterrupted PBR operation is possible. It also helps in the efficient utilization of fissile material due to which high burn-up can be achieved. Gaseous coolant, usually helium, moves from the top of the bed through the voids formed between the solids and removes fission heat generated from the heat source (Chapin et al. 2004; Khane et al. 2012; Khane 2014; Khane et al. 2016).

Granular materials (i.e., pebbles) exhibit solids-type behavior when at rest, whereas they exhibit partial fluid-type behavior when flowing (i.e., granular materials will flow from vessels under the influence of gravity but the mass flow rate will be approximately independent of the head of the 
material above it). This kind of behavior can be attributed to the friction between particles and between particles and the wall. Due to this complex behavior, there is still a lack of unified theory for granular materials. The dynamic core of a PBR is also a cause of concern for safety analyses and licensing, and thermal hydraulics. Hence, it is crucial to have full knowledge about pebbles flow field in terms of overall and zonal residence time distribution, velocities, and stagnant zones, if any. Conventional optics-based velocimetry techniques are of limited use for investigation of granular flow in a PBR, as these systems are dense and opaque. Hence, many of previous studies (Bazant 2004; Kadak et al. 2006; Li et al. 2009; Yang et al. 2009; Gui et al. 2014; Jia et al. 2014) were carried out using a half-model or $180^{\circ}$ model of actual PBR. Due to an additional transparent wall in such half-modes, actual granular flow is not very well mimicked. Particles at the mid-plane transparent wall were tracked visually and in an intermittent manner in such half-models. In some of previous studies (Gatt 1973; Gatt 1977; Bazant 2004; Shehata 2005), a collimated detector-based radioactive particle tracking technique was used to track the motion of pebbles in a scaled PBR model. These studies provided limited information about pebbles' residence time which is directly affected by the non-idealities associated with pebbles flow in the core such as pebble stagnation (Gui et al. 2014). Hence, there is still a need to study granular flow experimentally in order to provide full knowledge of pebble flow inside the reactor. This information is important for burn-up estimation, and devising re-fueling strategies for steady state core design.

The main objective of this research is to study slow and dense granular flow in a cold-flow recirculation experimental setup by using advanced noninvasive radioisotope-based techniques such as residence time distribution (RTD) and radioactive particle tracking (RPT). Both techniques have the same operational principal and both have no limitations from system opacity. These techniques can provide detailed information about pebble flow fields, overall and local residence time distribution of pebbles, stagnant zones and their sizes, and many other parameters (Al-Dahhan 2009). Also, they can provide further insights on non-idealities (i.e. stagnant zones) associated with pebbles flow in the core in a noninvasive manner. A stagnant/dead zone may exist in the pebble bed reactor near the transition from cylindrical to conical section at the pebbles outlet section for recirculation from the bottom of PBR as shown in Gatt's (1973, 1977) study or reported by DEM simulations (Rycroft et al. 2006; Yang et al. 2009; Khane et al. 2013; Khane 2014). Such investigations are of paramount importance and are 
required for basic reactor design calculations, estimation of fuel burn-up and core power distributions, devising refueling strategies, and safety analyses and assessments (Rycroft et al. 2006; Kadak 2007). In addition, these provided benchmark data are needed to validate computational methodologies associated with granular flows such as DEM commercial codes.

\section{Experimental setup}

The experimental setup mimics the slow flow of pebbles in the core under the influence of gravity with no gas flow. It has been reported that coarse particles are less affected by the fluid drag force than fine particles (Rao et al. 2008). Hence, there is no need to mimic downward flow of gaseous coolant through interstitial cavities for the investigation of slow and dense granular flow in a PBR. The pebble bed test reactor is made of acrylic material (1 foot outer diameter with 11.95 inch inside diameter and 1 foot in height). It is filled with $1 / 2$ inch diameter glass marbles as shown in Figure 2. Further details about determination of the important parameters such as: poisson ration, modulus of elasticity, friction coefficient, restitution coefficient can be found elsewhere (Khane 2014) which will be reported in a future manuscript. In pebble bed reactors, oscillatory variation of radial porosity is reported in many previous works and is observed up to five pebble diameters from the wall (Goodling et al. 1983; Mueller 1992; Mariani et al. 2009). Beyond five pebble diameters, there are minor fluctuations observed in the radial porosity. Hence, the effect of wall is not felt beyond five pebble diameters. Also, glass marbles of $1 / 2$ inch

diameter and $\sim 2.5 \mathrm{~g} / \mathrm{cm}^{3}$ density are found suitable to start with from tracer preparation and represent actual pebble flow (actual density of pebbles $\sim 1.8 \mathrm{~g} / \mathrm{cm}^{3}$ ). The diameter of the test reactor is selected based on considerations that representing the wall effect as the nature of packing will affect the subsequent flow of pebbles. Large diameter test reactors will have $\underline{\text { significant attenuation, thereby demanding a stronger radioactive source and are thus avoided in }}$ this work. One foot ( 12 inch) diameter of test reactor yields a diameter aspect ratio (which is defined as a ratio of inside cylinder diameter to the pebble diameter) of 23.9. Such an aspect ratio reduces the wall effect and is capable of introducing wall-effect induced oscillatory variation in radial porosity observed in an actual reactor (Al-Dahhan et al. 1995). An exit flow rate of one pebble every five seconds is chosen to represent slow granular flow in an actual pebble bed reactor. This flow rate was controlled by using a variable frequency operational solenoid valve connected at the pebbles exit as shown in Figure 3. A height-to-diameter ratio (H/D) of 2 or greater will yield long duration of radioactive particle tracking (RPT) experiments. There is a 
head independence of pressure and flow rate reported in previous studies related to a granular flow (McCabe et al. 1993), which is due to the static friction between the wall and the particles. As the coefficient of static friction increases, significantly higher head independence of pressure and flow rate is observed (Luo et al. 2010). Thus, a height-to-diameter ratio (H/D) of 1 is selected for this study. The bottom cone angle has significant influence on the flow of pebbles and presence of dead zones. During evolution of the exit control mechanism, it was found that the bottom cone angle also affects the jamming of pebbles in the bottom section. A half-cone angle of $60^{\circ}$ is chosen based on previous studies (Gatt 1973; Wang 2011) and found to be less prone to jamming problems. However, the setup and its key mechanical components can be scaled up to accommodate taller reactors with larger pebble diameter (up to 2.36 inch $\sim 6 \mathrm{~cm}$ pebbles diameter).

The glass marbles exit the setup through an opening in the extractor tube (via the rotation of smoothed-surface vane-type cup) without getting jammed and fall into a conveyor bin just below the reactor as shown in Figure 3. From there, the glass marble is transferred back to the hopper at the top via adjustable speed conveyor (TipTrak from UNITRAK). Conveyor bin releases glass marbles in this hopper. Marbles are then transferred to the top of the reactor via an inlet control mechanism which consists of straight and elbow sections of one pebble diameter tube shown in Figure 2. This inlet control mechanism also has three swivel joints. The inlet control mechanism, shown in Figure 4, is connected to a top plate (diameter matching with the reactor) with 17 holes as shown in Figure 5. These holes are provided to return the pebble at 17 different radial positions in a non-violent manner. The vertical leg of the conveyor belt is kept at a sufficient distance $(\sim 59$ inch $\sim 150 \mathrm{~cm})$ away from the test reactor.

As mentioned earlier, pebbles are returned in a non-violent matter (i.e., a returned pebble should not jump nor change its position). This is an important feature from RTD point of view and is required in actual PBR for implementation of devised refueling strategies for effective utilization of fissile material and other neurotic considerations such as power peaking related issues and flux flattening considerations. (Boer 2009). In addition, the setup and the design of its conveyor provide the needed space around the test reactor to implement the advanced noninvasive radioisotope-based flow visualization techniques for experimental investigation. 
(a)

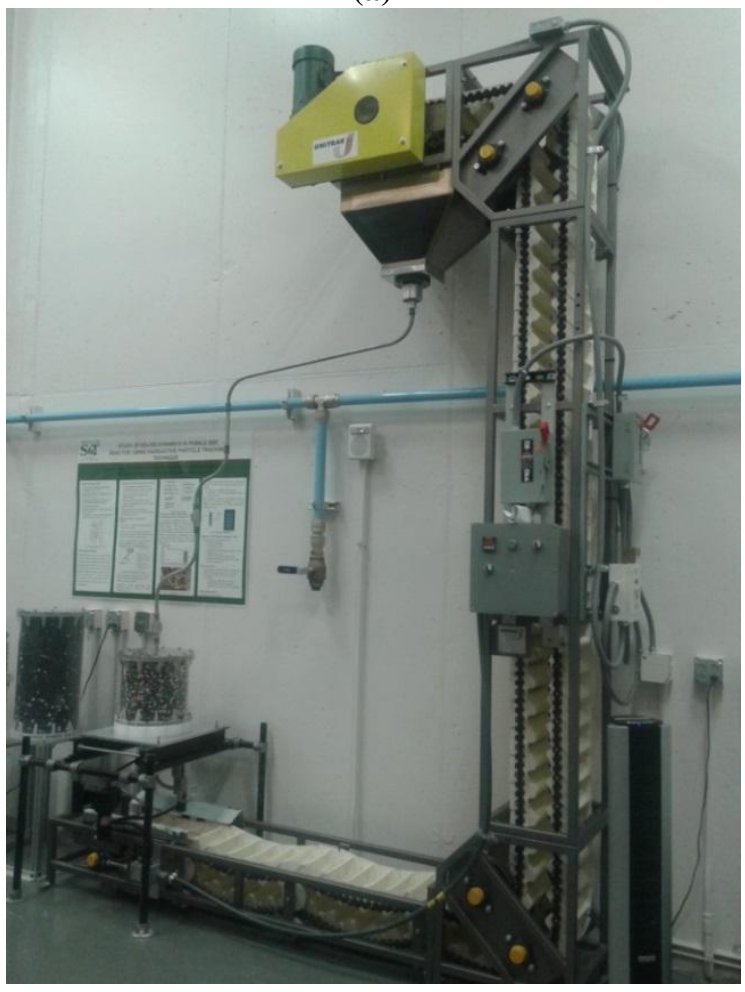

(b)

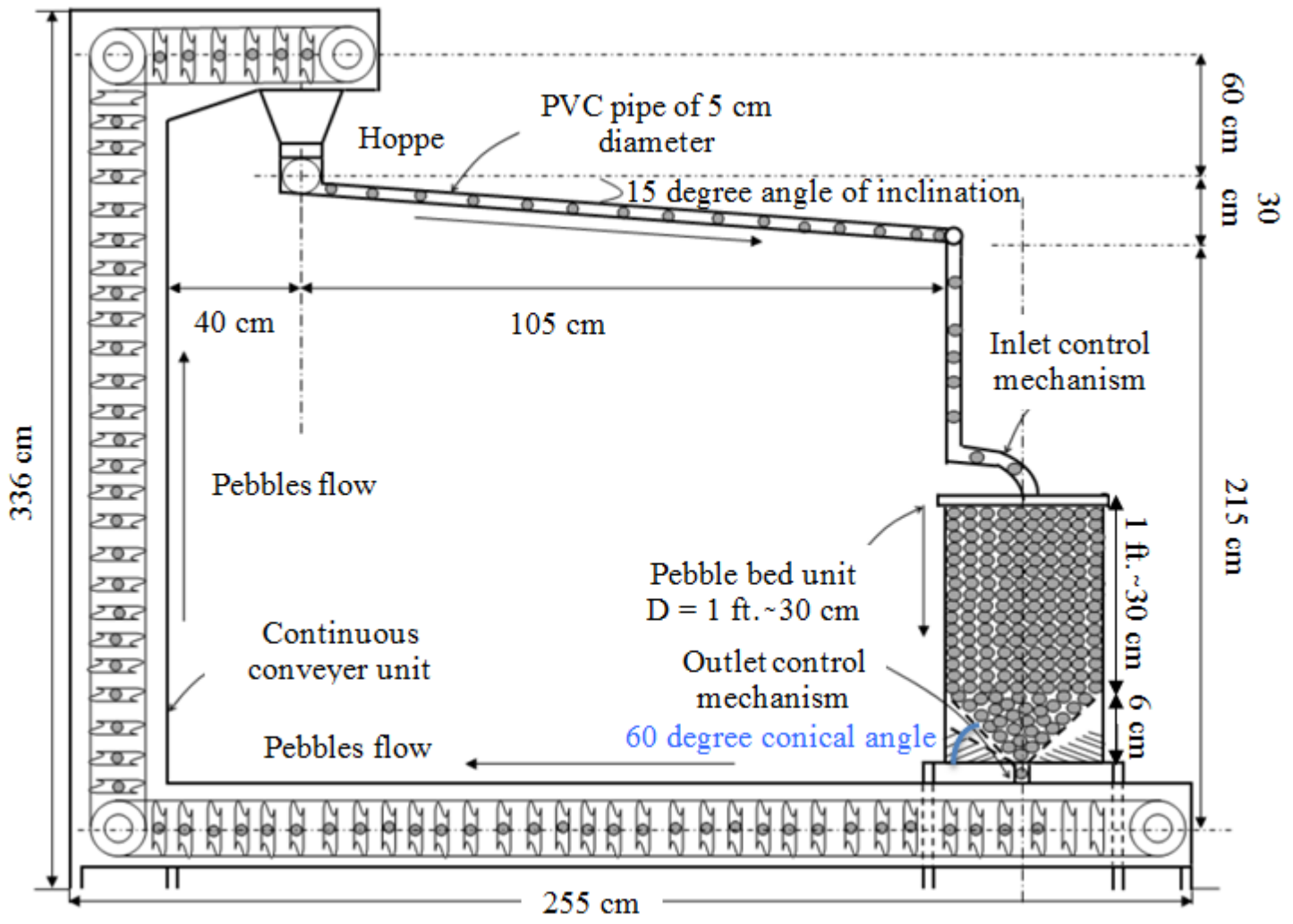

Figure 2: Continuous pebbles recirculation experimental setup (a) Pictorial representation and (b) Schematic diagram 
(a)

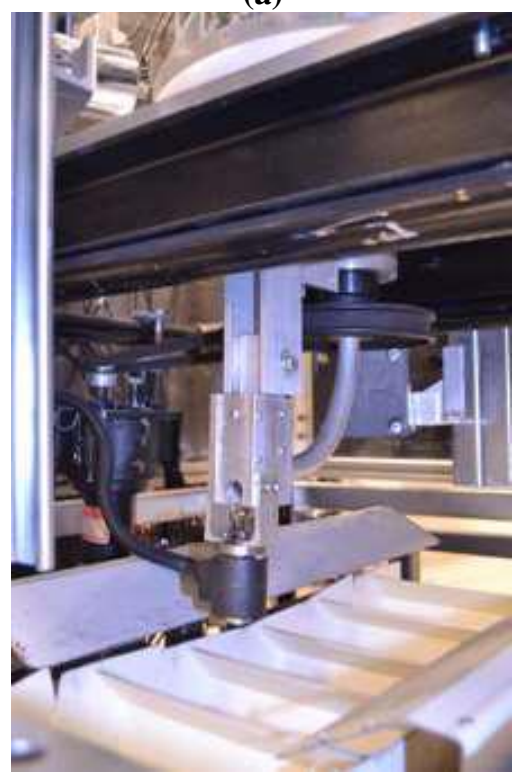

Figure 3: The outlet control mechanism (a) Pictorial representation and (b) Schematic diagram (b)

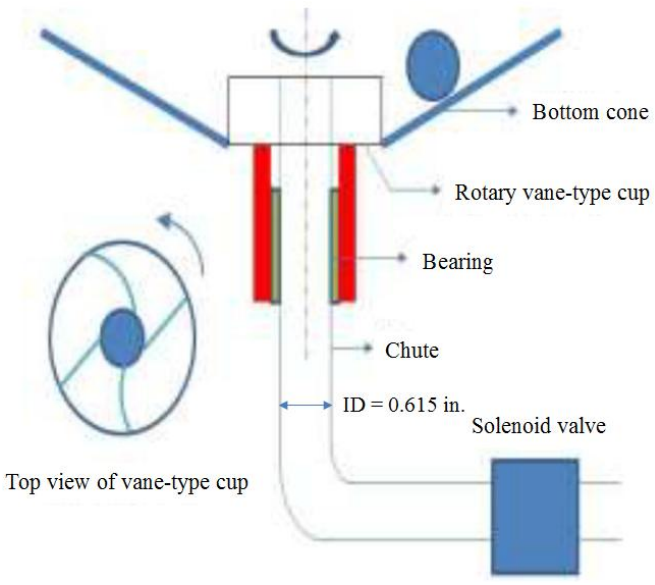

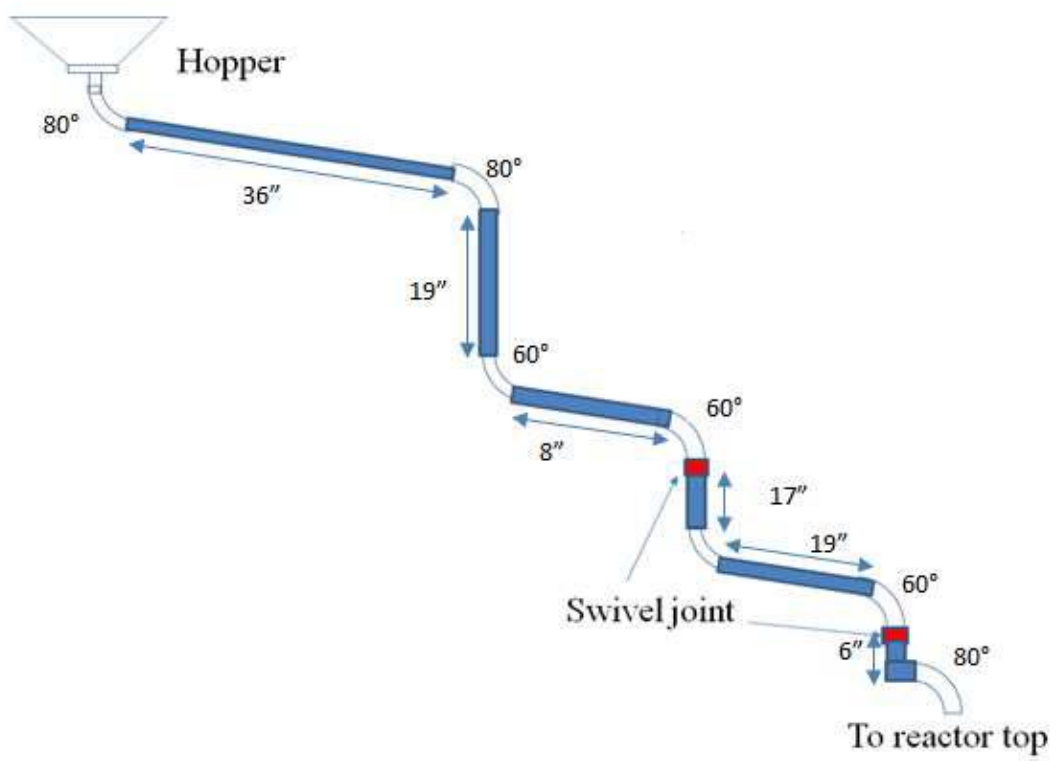

Figure 4: Schematic diagram of the inlet control mechanism 
(a)

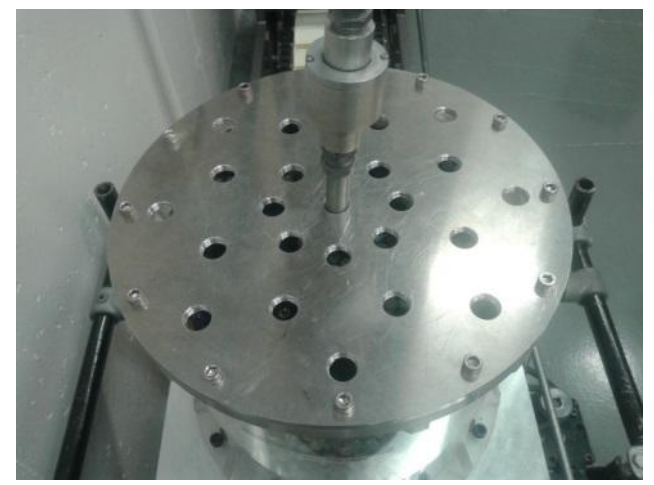

(b)

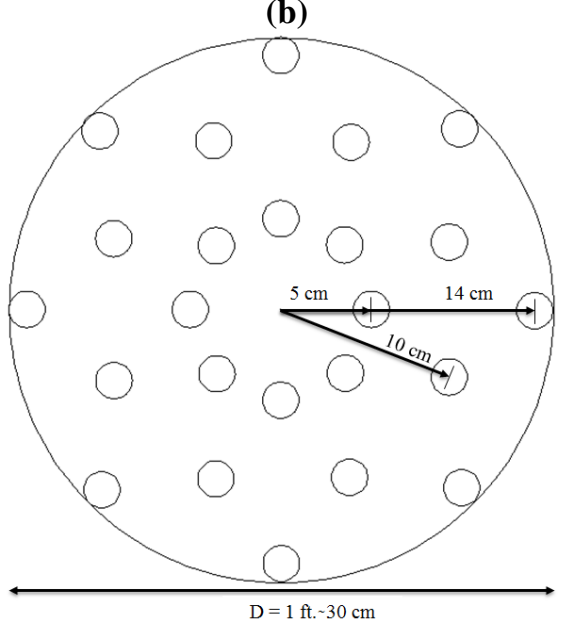

Figure 5: (a) Actual picture and (b) Schematic diagram of the top plate used for changing radial position of returned pebbles

\section{Description of the experimental work}

The time spent by pebbles at a particular position in the core is called local residence time while the time taken by pebbles from their entry into the core to its exit from the core is called global/overall residence time. The advanced RTD and RPT techniques are implemented to provide information about pebbles' global residence time inside the core, zonal residence time, and the z-component of average zonal velocity.

In the current study, the RTD of pebbles for different initial seeding positions of radioactive tracer is investigated to provide crucial information from burn-up estimation, devising re-fueling strategies and fuel mechanical damage (Shehata 2005; Boer 2009). Such a study can also provide further insight on flow non-idealities (deviation from ideal plug flow behavior), if any.

A dedicated RTD setup consisting of two collimated scintillation detectors was implemented around the continuous pebble recirculation experimental setup. One detector is located at the top entry of the pebble in a test reactor and the other one at the bottom near the reactor exit opening, as shown in Figure 6. The slit in the collimator is 2 inches $(\sim 5 \mathrm{~cm})$ in length, 1 inch $(\sim 2.5 \mathrm{~cm})$ thick and 0.039 inch $(\sim 1 \mathrm{~mm})$ in width, as shown in Figure 7. This setup is capable of measuring the pebbles' overall residence time in the test reactor in a noninvasive manner by monitoring the tracer particle motion inside the reactor. 


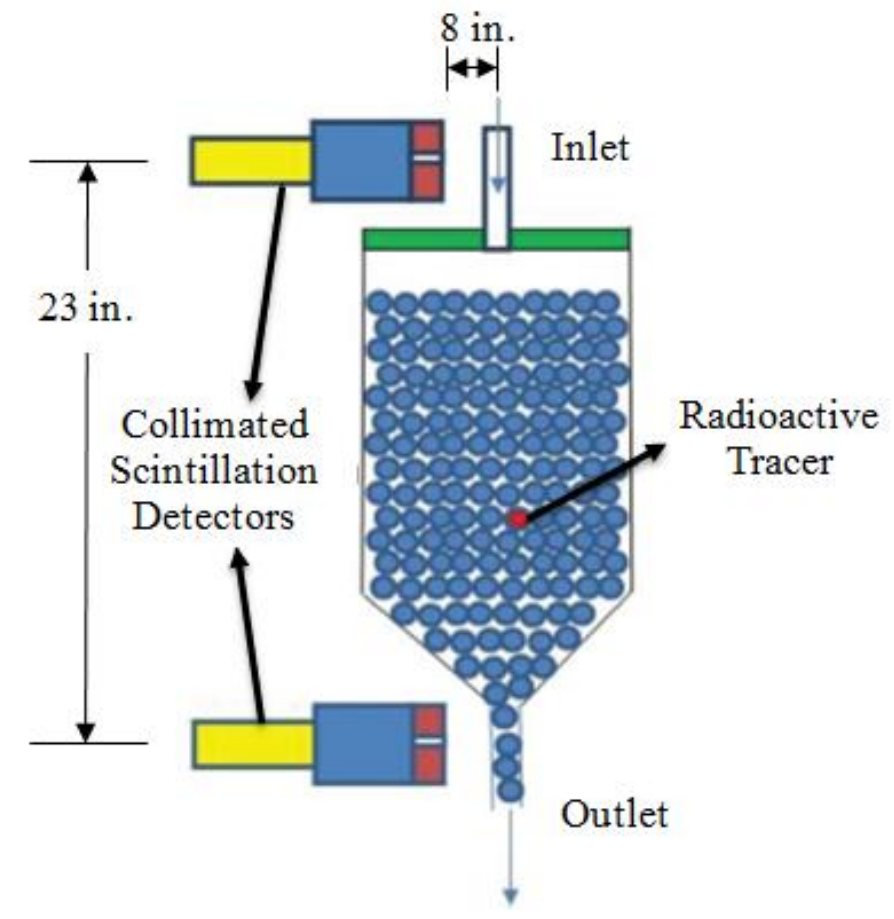

Figure 6: Schematic diagram of 2 detector based RTD setup

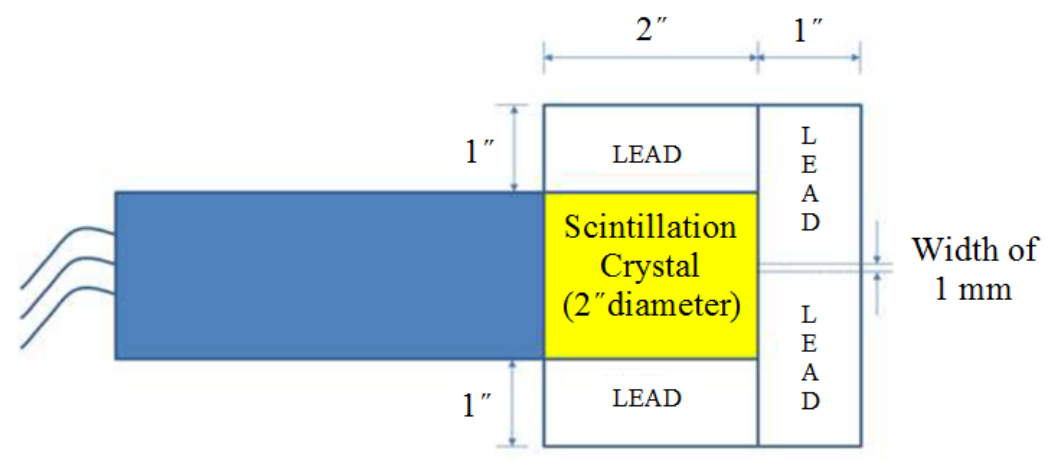

Figure 7: Lead collimator used in RTD detection

Using collimated detectors does not require any in-situ calibration to identify the instantaneous particle position of the tracer. The tracer position is identified by simultaneously monitoring photo-peak counts received by these detectors (Al-Dahhan 2009). In the current setup design, the detector collimators have horizontal slits. When the radioactive tracer particle is in the plane of the horizontal slit, maximum counts are recorded. This principle is used to record the time of entry and departure of the tracer particle from which overall residence time of pebbles is 
calculated for different initial seeding positions of the tracer particle. A sample of the counts response of top and bottom collimated detectors is shown in Figure 8.

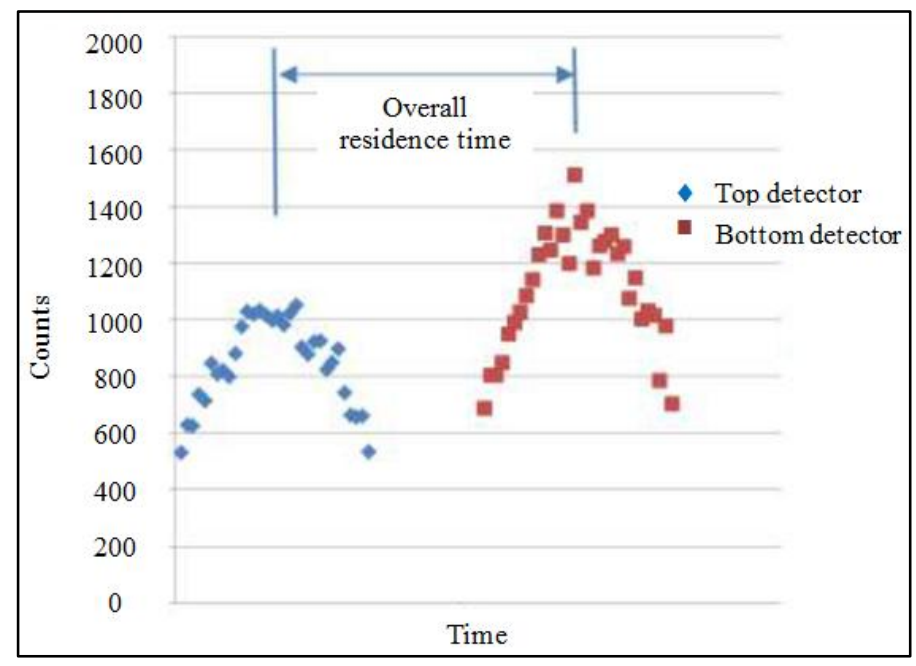

Figure 8: Counts response of top and bottom collimated detectors for RTD detection

On the other hand, determination of zonal residence time and z-component of average zonal velocities of tracer particles is executed by implementing a noninvasive RPT technique in which the pebbles' dynamics are investigated by monitoring the movement of a radioactive tracer particle by a set of non-collimated sodium iodide ( $\mathrm{NaI})$ scintillation detectors which are arranged strategically around the reactor (Moslemian et al. 1992; Al-Dahhan 2009).

The forward problem of finding instantaneous position of particle based on intensities received at the detectors is solved by performing calibration experiments (Khane et al. 2016). RPT calibration experiments, which are performed prior to actual RPT experiments, are carried out at the same operating conditions as that of actual experiment to mimic the radiation attenuation in the system (Khane 2014). During RPT calibration, a radioactive tracer particle is kept at known locations in the system and time-averaged counts data is recorded in all the detectors. An example of the obtained RPT calibration curves is shown in Figure 9. The generated calibration curves indicate that there is a spread in counts readings for the same tracer-detector distance. The instantaneous position of the tracer then can be found with the help of various in-house developed position reconstruction algorithms and calibration curves (Chen et al. 2001; Rados 2003; Vesvikar 2006; Han 2007; Shaikh 2007; Khane 2014). 


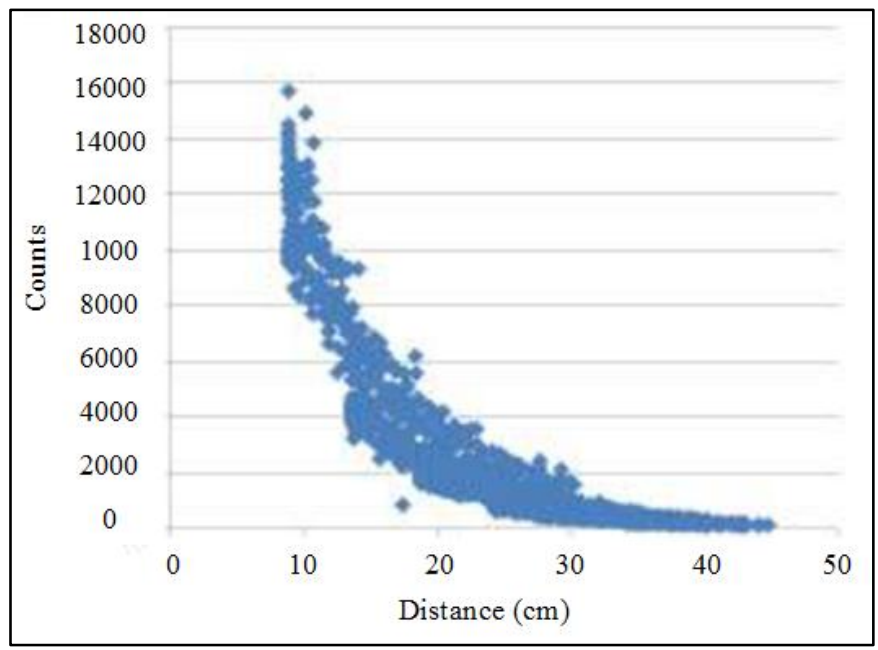

Figure 9: RPT detector calibration curve for PBR study

As mentioned earlier, the basic principle of noninvasive flow visualization techniques implemented in the current study is to monitor the movement of a tracer particle through the core. The main characteristic of a radioactive tracer is to mimic the dynamics of phase to be tracked. It should meet the following requirements:

- The density of the tracer should match the density of phase to be tracked.

- It should contain a suitable radioactive source of appropriate strength to ensure good measurement statistics (high signal to noise ratio) and should not saturate detectors. A longer half-life of the radioisotope is desirable to avoid decay correction and reasonable working life.

- When tracking a solid phase, the tracer should have the same size and shape as that solid, whereas it should be as small as possible for tracking the liquid phase to reduce drag forces.

- It should be rigid and thermally and mechanically stable at the operating conditions of the experiment

Generally, a tracer particle dynamically similar to the tracked phase is made-up of irradiated Scadium-46, Gold-198, Cobalt-60, or another isotope of a gamma ray emitter. As the source strength is reduced to half of its initial value in one half-life, the half-life of selected radionuclide/radioisotope should be an order of magnitude higher than that of the total duration of the given set of experiments. This will ensure that there is no significant reduction in the 
activity of source during the experiment. This is particularly important for long-lasting experiments such as the slow flow of pebbles in a PBR.

It is essential to have a source of sufficient strength for better statistics and reliable measurements. This will ensure that a high signal-to-noise ratio is observed even in the distant location of the source from the detector. At the same time, selected strength value should not saturate detectors when the source is very close to the detector. Based on these two opposing requirements, minimum radioactivity of the tracer particle for a given size of reactor and attenuating medium is decided. A strong source of radioactivity is required to study granular flow in a PBR due to the presence of highly attenuating glass marbles. A radioactive Co-60 source of $500 \mu \mathrm{Ci}$ strength was chosen for this PBR study due to its prolonged half-life $(\sim 5.2$ years) and lesser irradiation time requirement in a nuclear reactor. The Co-60 radioactive tracer particle (initial strength of $500 \mu \mathrm{Ci}$ ) is enclosed inside a Teflon tracer particle with the same shape, size, and density as that of $1 / 2$ inch diameter glass marbles shown in

Figure 10.

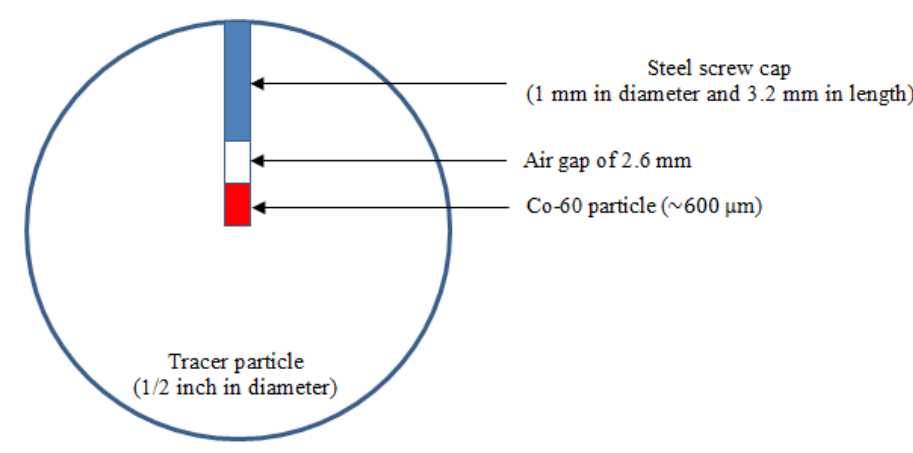

a. Schematic diagram

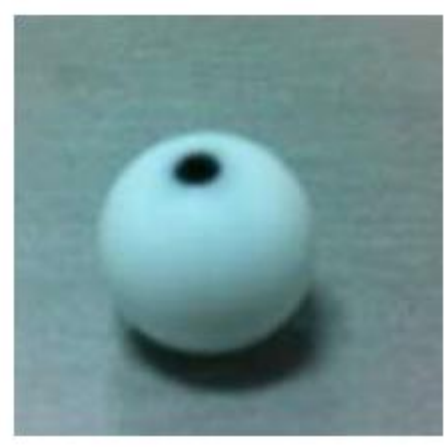

b. Pictorial representation

Figure 10: Co-60 tracer particle manufactured at Missouri S\&T labs 


\section{Results and discussion}

The effect of the initial seeding position of pebbles is investigated by carrying out experiments at different initial seeding positions of a radioactive tracer particle. The results for overall residence time for different initial seeding positions of a tracer are expressed in terms of overall residence time and transient number as shown in Figure 11 and Figure 12, respectively. The transient number is calculated based on the following equation (Gatt 1973, 1977):

Transient number $=\frac{\text { No. of pebbles recirculated between the seeding and and exit of the tracer }}{\text { total number of pebbles in the bed }}$

A transient number of 1 indicates that the whole bed inventory is recirculated between the initial seeding of the tracer and its exit. In Gatt's (1973) study, it was reported that transient number increases with increasing the seeding distance from the center of the bed. To check the effect of initial seeding radius, four experiments were carried out. Tracer was initially seeded at different dimensionless radial positions $(\mathrm{r} / \mathrm{R})$ of $0,0.33,0.67$ and 0.92 . It was found that overall residence time/transient number increases at a slower rate for dimensionless radial positions (r/R) between 0 and 0.33 , whereas it increases at a faster rate for dimensionless radial positions above (r/R) of 0.33 and is highest in a region close to the wall. This also suggests that there is a possibility of a faster moving zone of pebbles close to the center. Transient number for initial dimensionless seeding position (r/R) of 0.67 is found to be close to 1 . For particles between the initial dimensionless seeding position $(\mathrm{r} / \mathrm{R})$ of 0.67 and the outer periphery, more than one bed inventory needs to be recirculated before the tracer leaves the system. These findings are consistent with the Gatt's (1973) study and also consistent with the findings of computational simulations of Gui et al. (2014) that have shown that the radial RTD increases from about 50 seconds in the center region to about 80 seconds near the wall regions. 


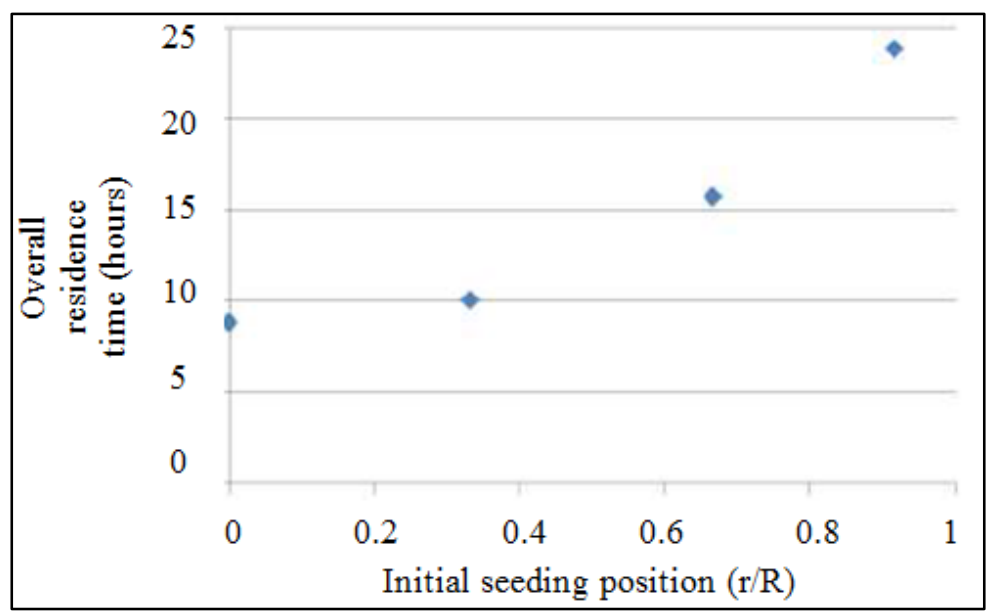

Figure 11: Overall pebbles residence time in hours

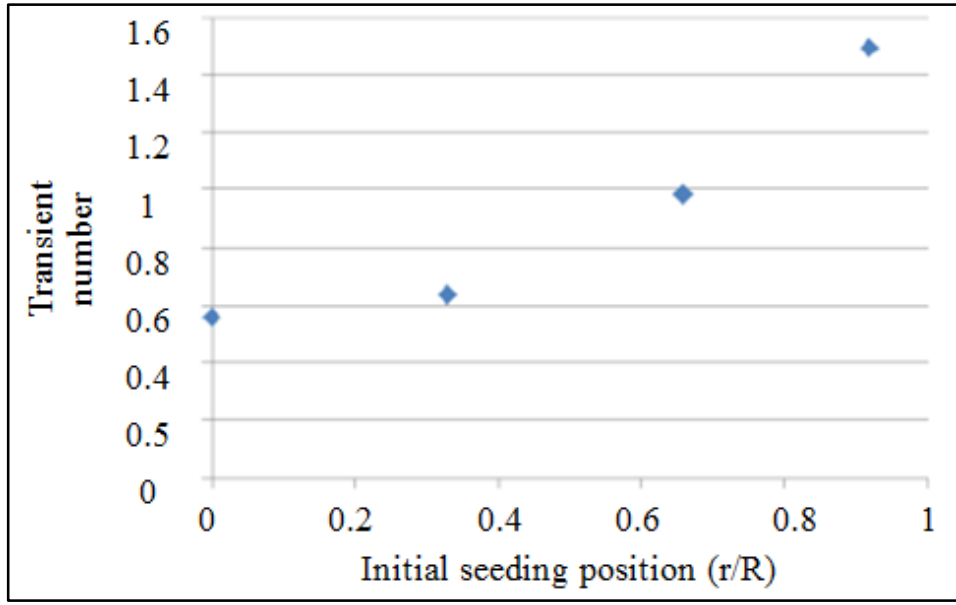

Figure 12: Overall pebbles residence time in terms of transient number

For more insights and understanding of the pebbles dynamics and different zones in the PBR core, the whole reactor was divided into three zones: Zone I (from the height of 10 to $20 \mathrm{~cm}$ ), Zone II (from the height of 20-30 cm) and Zone III (from the height of 30 to $36 \mathrm{~cm}$ ) as shown in Figure 13 (a). These zones were created to investigate zonal residence time and average $\mathrm{z}$ component of average zonal velocities. These parameters were investigated by employing the radioactive particle tracking (RPT) technique which uses the same principle as RTD technique in which the pebbles' dynamics are detected by monitoring the movement of a radioactive tracer particle by a set of non-collimated sodium iodide ( $\mathrm{NaI}$ ) scintillation detectors that are arranged strategically around the reactor (Moslemian et al. 1992; Al-Dahhan 2009; Khane et al. 2016). RPT results were analyzed to provide more information about residence time in different zones 
and average zonal velocities. The obtained results about zonal residence times are tabulated in Table 1 and are shown in Figure 13 (b) through (d).

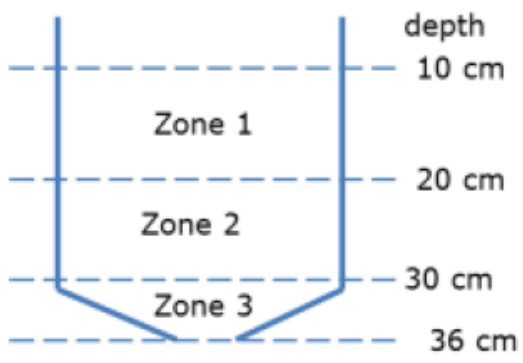

a. Zone-wise division of reactor

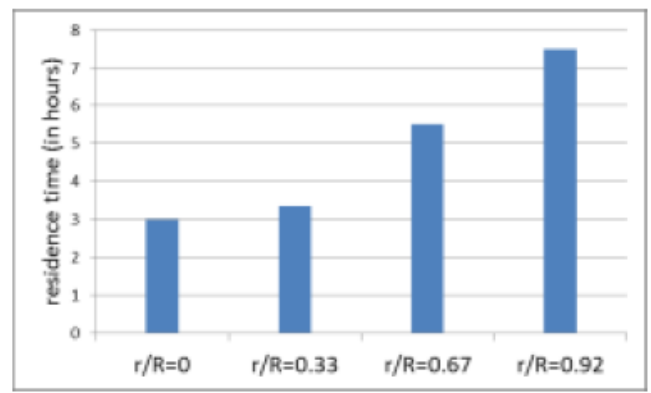

c. Zone -2 residence time

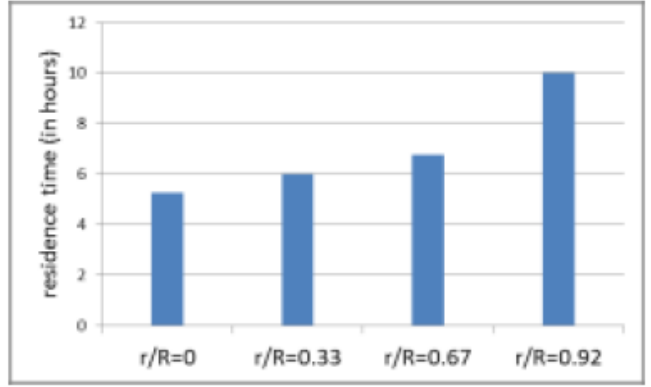

b. Zone -1 residence time

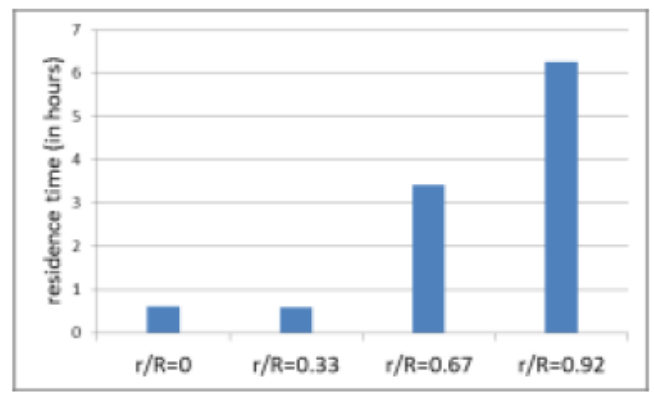

d. Zone-3 residence time

Figure 13: Zonal residence time results obtained using RPT technique

Table 1: Overall/Zonal residence times for different initial seeding positions of tracer (values in brackets represents percentage of overall residence time)

\begin{tabular}{c|c|c|c|c}
\hline & \multicolumn{4}{|c}{ Residence time in hours } \\
\hline & r/R = 0.0 & r/R= 0.33 & r/R = 0.67 & r/R = 0.92 \\
\hline Zone 1 & $5.25(59.3 \%)$ & $6(59.4 \%)$ & $6.75(43.1 \%)$ & $10(42.1 \%)$ \\
\hline Zone 2 & $3(33.9 \%)$ & $3.5(34.7 \%)$ & $5.5(35.1 \%)$ & $7.5(31.6 \%)$ \\
\hline Zone 3 & $0.6(6.8 \%)$ & $0.61(5.9 \%)$ & $3.42(21.8 \%)$ & $6.27(26.3 \%)$ \\
\hline $\begin{array}{c}\text { Overall increase } \\
\text { with respect to } \\
\text { shortest residence } \\
\text { time }\end{array}$ & $8.86(100 \%)$ & $10.1(100 \%)$ & $15.67(100 \%)$ & $23.77(100 \%)$ \\
\hline
\end{tabular}

It is found that zonal residence time for each zone increases with an increase in the value of dimensionless initial seeding position (i.e., from center towards wall). The RTD setup results for overall residence time of tracer/transient number for different initial seeding positions, shown in 
Figure 11 and Figure 12, exhibited the same trend. The values of zonal residence time are highest for dimensionless initial seeding position (r/R) of 0.92 among all initial seeding positions.

The zonal residence time as a percentage of overall residence time, as previously shown in Table 1, decreases from Zone 1 to Zone 2 and further from Zone 2 to Zone 3, for all seeding positions. Percent increase in zonal residence time for dimensionless initial seeding positions $(\mathrm{r} / \mathrm{R})$ of $0.33,0.67$, and 0.92 are calculated using corresponding zonal residence time for dimensionless initial seeding position $(\mathrm{r} / \mathrm{R})$ of 0 and are tabulated in Table 2 . The highest percentage increase ( 943\% increase) in zonal residence time was observed for the Zone 3 initial seeding position of (r/R) 0.92 .

Table 2: Percentage increase in zonal residence time values

\begin{tabular}{c|c|c|c}
\hline & \multicolumn{3}{|c}{ \% increase in zonal residence time with respect to initial seeding position } \\
r/R=0.0
\end{tabular}

Zonal residence times are indicative of average zonal velocities in respective zones. Lesser zonal residence time is an indication of higher average velocity in that respective zone and vice versa. Figure 14 represents the z-component of average zonal velocities for different initial seeding positions of the tracer calculated using the following equation.

$$
\mathrm{z}-\text { component of averge zonal velocities }=\frac{\text { tracer movement in } \mathrm{z}-\text { direction }}{\text { zonal residence time in hours }}
$$

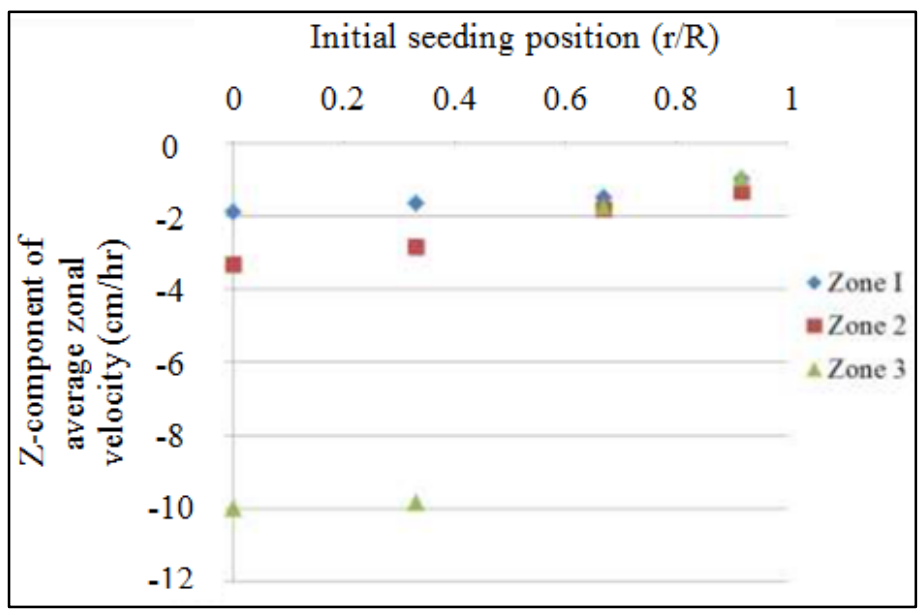

Figure 14: z-component of average zonal velocity for different initial seeding positions 
The smallest z-components of average zonal velocities, shown in Table 3, are observed in all the three zones for dimensionless initial seeding position $(\mathrm{r} / \mathrm{R})$ of 0.92 near the wall, whereas the highest z-components of average zonal velocities are observed in all the three zones for dimensionless initial seeding position $(\mathrm{r} / \mathrm{R})$ of 0 at the center. It is worth mentioning that the $\mathrm{z}-$ component of average zonal velocity is increasing from Zone 1 to Zone 2 and further from Zone 2 to Zone 3 for dimensionless initial seeding position (r/R) of 0 and 0.33 . For dimensionless initial seeding position (r/R) of 0.67 and 0.92 , it increases from Zone 1 to Zone 2 but decreases from Zone 2 to one 3.

Table 3: z-component of average zonal velocities for different initial seeding positions

\begin{tabular}{c|c|c|c|c|c}
\hline & \multicolumn{4}{|c|}{$\begin{array}{c}\text { Tracer initial seed position } \\
\text { (dimensionless radial position (r/R)) }\end{array}$} & \multirow{2}{*}{ Zone number } \\
\hline & $\mathbf{0 . 0}$ & $\mathbf{0 . 3 3}$ & $\mathbf{0 . 6 7}$ & $\mathbf{0 . 9 2}$ & \\
\hline \multirow{2}{*}{$\begin{array}{c}\text { z-component } \\
\text { of average } \\
\text { velocity } \\
(\mathbf{c m} / \mathbf{h r})\end{array}$} & 1.90 & 1.67 & 1.48 & 1.00 & Zone 1 \\
\cline { 2 - 5 } & 3.33 & 2.86 & 1.82 & 1.33 & Zone 2 \\
\cline { 2 - 5 } & 10.00 & 9.84 & 1.75 & 0.96 & Zone 3 \\
\hline
\end{tabular}




\section{Remarks}

The following are the highlights of the work carried out and the key findings of this study:

- Overall/zonal pebbles residence time distribution through a PBR is investigated by monitoring the motion of a radioactive tracer particle seeded at different initial seeding positions (r/R) of $0.33,0.67$, and 0.92 .

- Overall residence time/transient number increases with change in dimensionless initial seeding position $(\mathrm{r} / \mathrm{R})$ from the center towards the wall (169\% increase is observed for $\mathrm{r} / \mathrm{R}$ of 0.92 with respect to $\mathrm{r} / \mathrm{R}$ of 0 ).

- Zonal residence times are investigated and used to calculate average zonal velocities in respective zones. Smaller values of z-component of average zonal velocities are observed in all the three zones for initial seeding position (r/R) of 0.92 (close to the wall). On the other hand, larger values of z-component of average zonal velocities are observed in the three zones for an initial seeding position (r/R) of 0 (at the center).

- In Zone 3, the difference between z-components of average zonal velocities for initial seeding position close to the wall and at the center is more pronounced $(0.96 \mathrm{~cm} / \mathrm{hour}$ versus $10 \mathrm{~cm} /$ hour). This observation is consistent with previous observations reported in the PBMR safety analysis report.

- The average zonal velocity of tracer gradually increases from Zone 1 to 2 and further from Zone 2 to 3 for all the seeding positions.

- Radial movement of the tracer has been observed in Zone 2 and Zone 3 for all initial seeding positions except at the center. The highest radial movement of $9.36 \mathrm{~cm}$ in Zone 3 is observed for initial seeding position $(\mathrm{r} / \mathrm{R})$ of 0.92 .

- Residence time results provided benchmark data that could be used for assessment of commercial/in-house DEM-based simulation results. These validated computational methodologies can then be used to carry out high fidelity simulations and obtain predictions of actual scale PBR core dynamics. 


\begin{tabular}{l|l}
\hline \multicolumn{2}{l}{ Nomenclature } \\
\hline Symbol \\
\hline$r$ & Radial position of pebble seeding \\
\hline$R$ & Total radius of reactor \\
\hline$H$ & Experimental setup height \\
\hline$D$ & Experimental setup diameter \\
\hline Dimensionless groups \\
\hline$r / R$ & Dimensionless radial position \\
\hline List of abbreviations \\
\hline$G I F$ & The Generation IV International Forum \\
\hline$V H T R$ & Very High Temperature Reactor \\
\hline$N G N P S$ & Next Generation Nuclear Plants \\
\hline$P M R$ & Prismatic modular reactor \\
\hline$P B R$ & Pebble bed reactor \\
\hline$T R I S O$ & Tri-Structural Isotropic or Tri-Isotopes \\
\hline$D E M$ & Discrete element method \\
\hline$R T D$ & Residence time distribution \\
\hline$C F D$ & Computational fluid dynamics \\
\hline
\end{tabular}

\section{Acknowledgements}

The authors acknowledge the financial support provided by the Department of Energy (DOE) Nuclear Energy Research Initiative (NERI) project (NERI-08-043). 


\section{References}

Al-Dahhan, M. H. (2009). "Radioisotopes applications in industry: an overview." Atoms for Peace: International Journal 2(4): 324.

Al-Dahhan, M. H., Y. Wu and M. P. Duduković (1995). "Reproducible technique for packing laboratory-scale trickle-bed reactors with a mixture of catalyst and fines." Industrial and Engineering Chemistry Research 34(3): 741-747.

Bazant, A. C. K. a. M. Z. (2004). Pebble flow experiments for pebble bed reactors. 2nd International Topical meeting on High Temperature Reactor Technology. Beijina, China.

Boer, B. (2009). Optimized core design and fuel management of a pebble-bed type nuclear reactor. Ph.D Thesis, University of Technology, Delft.

Chapin, D., S. Kiffer and J. Nestell (2004). "The Very High Temperature Reactor: A Technical Summary." MPR Associates, Inc.

Chen, J., N. Rados, M. H. Al-Dahhan, M. P. Duduković, D. Nguyen and K. Parimi (2001). "Particle motion in packed/ebullated beds by CT and CARPT." AIChE Journal 47(5): 994-1004.

Curtius, H., G. Kaiser, N. Lieck, M. Güngör, M. Klinkenberg and D. Bosbach (2015). "Spent UO2 TRISO coated particles - Instant release fraction and microstructure evolution." Radiochimica Acta 103(6): 433-442.

Gatt, F. (1973). "Flow of spheres and near spheres in cylindrical vessels, part IV." Australian Energy commission, Lucas Heights.

Gatt, F. C. (1977). "Flow of individual pebbles in cylindrical vessels." Nuclear Engineering and Design 42(2): 265-275.

Goodling, J. S., R. I. Vachon, W. S. Stelpflug, S. J. Ying and M. S. Khader (1983). "Radial porosity distribution in cylindrical beds packed with spheres." Powder Technology 35(1): 23-29.

Gui, N., X. Yang, J. Tu and S. Jiang (2014). "Effect of bed configuration on pebble flow uniformity and stagnation in the pebble bed reactor." Nuclear Engineering and Design 270: 295301.

Han, L. (2007). Hydrodynamics, back-mixing, and Mass Transfer in a Slurry Bubble Column Reactor for Fischer-Tropsch Alternative Fuels. PhD Thesis, Washington University, Saint Louis, MO.

Jia, X., X. Yang, N. Gui, Y. Li, J. Tu and S. Jiang (2014). "Experimental and numerical study of stagnant zones in pebble bed." Science and Technology of Nuclear Installations 2014.

Kadak, A. C. (2007). "MIT pebble bed reactor project." Nuclear Engineering and Technology 39(2): 95-102.

Kadak, A. C. and M. V. Berte (2006). "Advanced modularity design for the MIT pebble bed reactor." Nuclear Engineering and Design 236(5-6): 502-509.

Khane, V. and M. H. Al-Dahhan (2013). Experimental and computational study of slow and dense granular flow in a pebble bed reactor. Transactions of the American Nuclear Society.

Khane, V. and D. Muthanna Al (2012). Study of pebbles residence time distributions in a pebble bed test reactor. Transactions of the American Nuclear Society.

Khane, V., I. A. Said and M. H. Al-Dahhan (2016). "Experimental investigation of pebble flow dynamics using radioactive particle tracking technique in a scaled-down Pebble Bed Modular Reactor (PBMR)." Nuclear Engineering and Design 302, Part A: 1-11.

Khane, V. B. (2014). Experimental and computational investigation of flow of pebbles in a pebble bed nuclear reactor. Ph.D Thesis, Missouri University of Science and Technology.

LaBar, M. P., A. S. Shenoy, W. A. Simon and E. M. Campbell (2004). "Introducing the GTMHR." Nuclear Engineering International 49(596): 18-23. 
Li, Y., Y. Xu and S. Jiang (2009). "DEM simulations and experiments of pebble flow with monosized spheres." Powder Technology 193(3): 312-318.

Luo, X., X. Li and S. Yu (2010). "Nuclear graphite friction properties and the influence of friction properties on the pebble bed." Nuclear Engineering and Design 240(10): 2674-2681.

Mariani, N. J., W. I. Salvat, A. Campesi, G. F. Barreto and O. M. Martínez (2009). "Evaluation of structural properties of cylindrical packed beds using numerical simulations and tomographic experiments." International Journal of Chemical Reactor Engineering 7.

McCabe, W. L., J. C. Smith and P. Harriott (1993). Unit operations of chemical engineering, McGraw-Hill New York.

Moses, D. L. (2010). Very High-Temperature Reactor (VHTR) Proliferation Resistance and Physical Protection (PR\&PP) ORNL.

Moslemian, D., N. Devanathan and M. P. Dudukovic (1992). "Radioactive particle tracking technique for investigation of phase recirculation and turbulence in multiphase systems." Review of Scientific Instruments 63(10): 4361-4372.

Mueller, G. E. (1992). "Radial void fraction distributions in randomly packed fixed beds of uniformly sized spheres in cylindrical containers." Powder Technology 72(3): 269-275.

Rados, N. (2003). Slurry bubble column hydrodynamics. PhD Thesis, Washington University, Saint Louis, MO.

Rao, K. K. and P. R. Nott (2008). An introduction to granular flow.

Rycroft, C. H., G. S. Grest, J. W. Landry and M. Z. Bazant (2006). "Analysis of granular flow in a pebble-bed nuclear reactor." Physical Review E - Statistical, Nonlinear, and Soft Matter Physics 74(2).

Shaikh, A. (2007). Bubble and Slurry Bubble Column Reactors: Mixing, Flow Regime Transition and Scaleup. PhD Thesis, Washington University, Saint Louis, MO.

Shehata, A. H. (2005). A new method for radioactive particle tracking Ph.D Thesis, North Carolina University.

Vesvikar, M. S. (2006). Understanding the hydrodynamics and performance of anaerobic digesters. PhD Thesis, Washington University, Saint Louis, MO.

Wang, Z. (2011). A dual measurement system for radioactive tracer pebble tracking in PBRs. $\mathrm{PhD}$ Thesis, North Carloina State University.

Weil, J. (2001). "Pebble-bed design returns." IEEE Spectrum 38(11): 37-40.

Yang, X., W. Hu and S. Jiang (2009). "Experimental Investigation on feasibility of two-regiondesigned pebble-bed high-temperature gas-cooled reactory." Journal of Nuclear Science and Technology 46(4): 374-381. 